\title{
Landscape heterogeneity strengthens the relationship between $\beta$-diversity and ecosystem function
}

\author{
Edd Hammill iD,${ }^{1,5}$ Charles P. Hawkins, ${ }^{1}$ Hamish S. Greig, ${ }^{2}$ Pavel Kratina, ${ }^{3}$ \\ Jonathan B. Shurin, ${ }^{4}$ and Trisha B. Atwood ${ }^{1}$ \\ ${ }^{1}$ Department of Watershed Sciences and the Ecology Center, Utah State University, 5210 Old Main Hill, Logan, Utah 84322 USA \\ ${ }^{2}$ School of Biology and Ecology, 5751 Murray Hall, Orono, Maine 04469 USA \\ ${ }^{3}$ School of Biological and Chemical Sciences, Queen Mary University of London, London E1 4NS UK \\ ${ }^{4}$ Division of Biological Sciences, University of California, 9500 Gilman Dr., La Jolla, California 92093 USA
}

\begin{abstract}
Consensus has emerged in the literature that increased biodiversity enhances the capacity of ecosystems to perform multiple functions. However, most biodiversity/ecosystem function studies focus on a single ecosystem, or on landscapes of homogenous ecosystems. Here, we investigate how increased landscape-level environmental dissimilarity may affect the relationship between different metrics of diversity $(\alpha, \beta$, or $\gamma)$ and ecosystem function. We produced a suite of simulated landscapes, each of which contained four experimental outdoor aquatic mesocosms. Differences in temperature and nutrient conditions of the mesocosms allowed us to simulate landscapes containing a range of within-landscape environmental heterogeneities. We found that the variation in ecosystem functions was primarily controlled by environmental conditions, with diversity metrics accounting for a smaller (but significant) amount of variation in function. When landscapes were more homogeneous, $\alpha, \beta$, and $\gamma$ diversity was not associated with differences in primary production, and only $\gamma$ was associated with changes in decomposition. In these homogeneous landscapes, differences in these two ecosystem functions were most strongly related to nutrient and temperature conditions in the ecosystems. However, as landscape-level environmental dissimilarity increased, the relationship between $\alpha$, $\beta$, or $\gamma$ and ecosystem functions strengthened, with $\beta$ being a greater predictor of variation in decomposition at the highest levels of environmental dissimilarity than $\alpha$ or $\gamma$. We propose that when all ecosystems in a landscape have similar environmental conditions, species sorting is likely to generate a single community composition that is well suited to those environmental conditions, $\beta$ is low, and the efficiency of diversity-ecosystem function couplings is similar across communities. Under this low $\beta$, the effect of abiotic conditions on ecosystem function will be most apparent. However, when environmental conditions vary among ecosystems, species sorting pressures are different among ecosystems, producing different communities among locations in a landscape. These conditions lead to stronger relationships between $\beta$ and the magnitude of ecosystem functions. Our results illustrate that abiotic conditions and the homogeneity of communities influence ecosystem function expressed at the landscape scale.
\end{abstract}

Key words: beta-diversity; decomposition; ecosystem function; ecosystem productivity; environmental heterogeneity; landscape ecology; macroinvertebrates; metacommunities.

\section{INTRODUCTION}

Human activities have dramatic impacts on the abiotic conditions and biota of natural ecosystems (Vitousek et al. 1997, Cardinale et al. 2012a). Atmospheric pollutants have affected global temperatures (Forster et al. 2007), while a shift towards industrial-scale agriculture has accelerated levels of nutrient release (Tilman 1999, Bouwman et al. 2009), leading to increased rates of eutrophication (Dupas et al. 2015). The realized impacts of changes in temperature and nutrient levels are not uniformly distributed across time and space, increasing heterogeneity in abiotic conditions among ecosystems within a landscape and producing novel environmental conditions (Ashcroft et al. 2009, Hobbs et al. 2009). Moreover, the dispersal of nutrient pollutants within a landscape varies considerably depending on soil type, hydrology, land use, and topography (Heathwaite et al. 2005, Okubo and Levin 2013). Human activities may

Manuscript received 2 March 2018; revised 16 July 2018; accepted 23 July 2018. Corresponding Editor: Evan L. Preisser.

${ }^{5}$ E-mail: edd.hammill@usu.edu therefore increase heterogeneity in temperature and nutrient conditions (hereon termed "environmental dissimilarity") above levels generated by natural processes. These changes to environmental conditions may directly alter rates of ecosystem functions (Gruber and Galloway 2008, Shurin et al. 2012), but also present critical challenges for understanding biodiversity/ecosystem function relationships at the landscape scale.

Biodiversity varies among and within ecosystems on every spatial and temporal scale (Barbour et al. 1999, Bailey et al. 2004, Resh 2007, Lefcheck et al. 2015), and its measure is both scale- and question-dependent. Local diversity, the number of species occurring within a local ecosystem, is usually referred to as $\alpha$-diversity (hereon $\alpha$ ). Landscape-level or regional diversity (i.e., the number of species observed across individual ecosystems) is termed $\gamma$-diversity (hereon $\gamma$ ). Turnover among communities within a region (differences in community composition or $\alpha$ among local ecosystems) has been broadly referred to as $\beta$-diversity (hereon $\beta$, Whittaker 1972).

There is growing consensus among ecologists that higher biodiversity often begets increased levels of ecosystem 
functioning across many types of ecosystems (Cardinale et al. 2012a). In addition, the specific species present, particularly the functionally dominant species, can have major impacts on ecosystem function (Grime 1998, Naeem et al. 2012, Atwood et al. 2014a). However, with a few notable exceptions (Pasari et al. 2013, Barnes et al. 2016, Burley et al. 2016, Hautier et al. 2018, Winfree et al. 2018), the majority of empirical investigations of biodiversity-ecosystem function relationships have focused on local species richness $(\alpha)$. This focus on local species richness overlooks the scale dependence of the biodiversity-ecosystem-function relationship (Isbell et al. 2017), and assumes that the processes driving the relationship are equal at the ecosystem and landscape level.

Within a single ecosystem, or a homogeneous landscape of abiotically similar ecosystems, species sorting may lead to a single, "functionally optimal" community, composed of taxa with a series of traits and population densities that are well suited to exploit available resources. Furthermore, theory suggests that community composition should be similar across such landscapes because the available niches ought to be filled by the same species in each ecosystem (Kraft et al. 2008). In this case, we would therefore expect that $\alpha$ would be similar among ecosystems, and both $\beta$ and $\gamma$ should be relatively low. In contrast, as environmental conditions across landscapes differ, species sorting should result in local communities that differ in species composition (Barnes et al. 2016). Each of these different communities then consists of species best adapted to each local habitat (Winder et al. 2009 ), and potentially meaning that species turnover $(\beta)$ is more important to maintain ecosystem function than $\alpha$ at larger spatial scales (Winfree et al. 2018). We therefore expect that, as we move from the ecosystem scale to the landscape scale, environmental heterogeneity within a landscape may mean there is no single, functionally optimal community that is able to best perform ecosystem functions (Isbell et al. 2017, Winfree et al. 2018). In landscapes with a high degree of environmental heterogeneity, we might observe similar levels of $\alpha$ if each ecosystem provides a similar amount of niche space, but higher $\beta$ and $\gamma$ than in homogeneous landscapes. Moreover, higher levels of $\beta$ may be associated within increased ecosystem functioning in heterogeneous landscapes, as different communities may perform different functions, and perform some functions to a greater degree, under different environmental conditions (Thompson and Gonzalez 2016, Isbell et al. 2017). In addition, changes to abiotic conditions may increase the importance of stochastic determinants of community composition (Chase 2010), leading to changes in $\alpha, \beta$, and $\gamma$ and ecosystem functions either directly (Burley et al. 2016) or indirectly (Barnes et al. 2016).

To understand how differences in landscape-level environmental dissimilarity can alter the relationships among ecosystem functions and $\alpha, \beta$, and $\gamma$, we used data from an outdoor freshwater mesocosm experiment in which invertebrate community composition and core ecosystem functions were measured. Temperature and nutrient conditions were manipulated, and the subsequent variation in conditions among mesocosms was used to create simulated landscapes with differing levels of environmental dissimilarity. This approach allowed us to examine how the relationships between ecosystem functions and $\alpha, \beta$, and $\gamma$ varied with environmental dissimilarity. For each mesocosm, we measured two ecosystem functions that represent basal trophic resources in aquatic food webs: primary production and leaf litter decomposition. We predicted that much of the variation in these ecosystem functions would be controlled by nutrient and temperature levels in the landscape due to the importance of bottom-up control, especially the role of nutrients in controlling primary production (Harpole et al. 2011). With respect to the role of diversity, we hypothesized that at low levels of environmental dissimilarity, $\alpha$ and $\gamma$ would be more strongly associated with landscape-level ecosystem functions than $\beta$. In this case, the absolute diversity within both individual ecosystems and the landscape as whole would influence landscape-level ecosystem function, and a single functionally optimal community would occupy all habitats within the landscape. However, at higher levels of environmental dissimilarity, we hypothesized that $\beta$ would be more strongly associated with landscape-level ecosystem functions than either $\alpha$ or $\gamma$. When environmental conditions among ecosystems in a landscape differ, each ecosystem within the landscape has the potential to have a different community that is well adapted to the particular abiotic conditions of that ecosystem.

\section{Methods}

\section{Experimental set-up and data collection}

Experimental ponds consisted of 20, 1136-L, Rubbermaid (Sandy Springs, Georgia, USA) cattle water tanks housed at the University of British Columbia's pond facility (Vancouver, British Columbia, Canada) and represented a subset of treatments from a broader experiment (Greig et al. 2012, Kratina et al. 2012). This broader experiment contained mesocosms where a top predator had been added, and we elected to exclude these treatments from the current analysis to focus on the effects of nutrients and temperature. Nutrient and temperature conditions among the ponds were directly manipulated in a $2 \times 2$ fully factorial design. Nutrient levels within each pond were designated as either ambient or elevated, with elevated conditions being achieved through the monthly additions of $264 \mu \mathrm{g}$ of nitrogen/L (as $\mathrm{NaNO}_{3}$ ) and $27 \mu \mathrm{g}$ of phosphorus/L (as $\mathrm{KH}_{2} \mathrm{PO}_{4}$ ). Temperature treatments were designated as either ambient or elevated. Heating tanks with $300 \mathrm{~W}$ Hagen aquarium heaters (Hagen, Montreal, Canada) produced temperatures $3^{\circ} \mathrm{C}$ above ambient tank temperatures. Nutrient and warming treatments were randomly assigned to the 20 ponds, to generate four experimental treatment combinations of five replicates each. At the start of the experiment, mesocosms were inoculated with phytoplankton, zooplankton, macroinvertebrates, and sediments from local sources. The experimental conditions were maintained for 12 months, during which time the tanks were open to allow natural colonization of macroinvertebrates and plankton from local sources. We assume that, due to the close spatial proximity of the mesocosms ( $1 \mathrm{~m}$ between mesocosms), there were minimal differences in dispersal rates. This lack of differential dispersal rates, together with the fact that identical communities were initially inoculated, would mean that differences in diversity 
were generated predominantly through species sorting under the different treatment conditions within the mesocosm, and stochastic processes. After 12 months, abundance data on 25 benthic macroinvertebrates and 16 zooplankton taxa were used to calculate $\alpha, \beta$, and $\gamma$ diversity of consumers. Benthic macroinvertebrates were sampled with two $0.02-\mathrm{m}^{2}$ cylinder pipe samples per tank along with quantitative wall and water column sweeps, whereas zooplankton were collected in a 10-L depth-integrated water column samples (details in Kratina et al. 2012, Shurin et al. 2012). We also collected data on two ecosystem functions: leaf litter decomposition and net ecosystem productivity (NEP). Decomposition rates were calculated by fitting negative exponential decay models to the mass of leaf matter (bigleaf maple, Acer macrophyllum) present in 10-mm mesh Nitex (Dynamic Aqua-Supply Ltd, Surrey, Canada) bags at 0 and 8 weeks (Greig et al. 2012). Net ecosystem productivity was calculated as the difference in $\mathrm{O}_{2}$ concentrations between dawn and dusk for each mesocosm (Shurin et al. 2012). This metric of NEP therefore measures the difference between photosynthesis and respiration of the whole community over the course of the daylight hours.

\section{Simulated landscapes}

The 20 experimental ponds were arranged in all possible combinations to construct 4,845 unique simulated landscapes. These simulated landscapes were generated by randomly selecting a unique, random sample without replication of 4 of the 20 ponds (Pasari et al. 2013). As there was no spatial component to the experiment, the ecosystems placed into each experimental landscape were selected independently of their spatial location. Environmental heterogeneity within the landscape was indexed using a $2 \times 2$ matrix of environmental conditions. Each experimental pond within the landscape was classified according to its environmental conditions, that is, whether its nutrient levels were ambient (1) or elevated (2) and whether temperature was ambient (1) or elevated (2). The environmental dissimilarity index was then calculated as the Manhattan distance between the ecosystems.

The number of potential ecosystem combinations that can produce landscapes with different environmental dissimilarities is substantially different (e.g., there are 20 different mesocosm combinations that produce landscapes with a dissimilarity of 0 but 2,000 combinations produce a dissimilarity of 7). To overcome this difference, we resampled simulated landscapes within each level of environmental dissimilarity 2,000 times to balance the number of landscapes across dissimilarity levels. The experimental design includes two levels of temperature and two levels of nutrients. Although changes in the two factors are unlikely to be exactly biologically equivalent, both increased nutrients and increased temperature represent ecosystem-level stressors that may generate pools of tolerant species that differ from communities under ambient conditions. Note that environmental dissimilarity index values of 1,2 , and 5 are mathematically impossible with this design.

For each simulated landscape, we calculated the landscape-level values of $\alpha, \beta$, and $\gamma$. Many previous investigations into the relationships between biodiversity and ecosystem functions have utilized biodiversity metrics based on species richness, which gives equal weighting to both rare and common species (Loreau et al. 2001, Cardinale et al. 2006, Lefcheck et al. 2015). However, several studies have suggested that using abundance-weighted biodiversity metrics are more robust as they are less impacted by incomplete sampling (Beck et al. 2013), and they may have greater impact on ecosystem function because they contain information about functional dominants (Barwell et al. 2015, Winfree et al. 2018). We calculated biodiversity metrics using both abundance-weighted and species richness and found that the results were largely in agreement for NEP, but differed slightly for decomposition (see Appendix S1). Because abundance-weighted metrics explained more of the variability in our ecosystem functions we focus our results and discussion on metrics calculated using abundanceweighting and provide the species richness results in a supplementary section (Appendix S1). The total diversity $(\gamma)$ was partitioned into $\alpha$ and $\beta$ components by decomposing the Shannon entropy (Jost 2007, 2010). This method of partitioning means that $\alpha$ and $\beta$ components are independent of each other and can then be converted into their numbers equivalents (or true diversity) by taking the exponential value of each metric (Jost 2007). As this method generates $\alpha$ and $\beta$ diversities that are independent of each other (Jost 2007), they can be included as uncorrelated variables within the statistical analyses. All these processes were performed using the entropart package in $\mathrm{R}$ programming language (Marcon and Hérault 2015).

\section{The relationships between environmental dissimilarity, diversity, and ecosystem functions}

To quantify how environmental dissimilarity affected each of $\alpha, \beta$, and $\gamma$, we regressed each diversity type against the environmental dissimilarity index. As previous experiments have demonstrated relationships between biodiversity and both temperature and nutrient levels (Worm et al. 2002, Wang et al. 2009), the number of mesocosms that had elevated nutrients or elevated temperature was included as covariates. The environmental dissimilarity index is independent of the number of elevated nutrient or temperature mesocosms as there are multiple ways to produce different values of the index. However, landscapes with an environmental dissimilarity of 8 always contained two mesocosms with elevated nutrients and two with elevated temperature crossed in a $2 \times 2$ manner, hence nutrient and temperature levels were completely redundant in landscapes with environmental dissimilarities of 8 . We therefore excluded temperature and nutrient covariates from these regressions.

We further determined how two ecosystem functions (NEP and leaf litter decomposition) were associated with $\alpha$, $\beta$, and $\gamma$. As NEP was estimated as the difference between photosynthesis (of both phytoplankton and periphyton) and respiration (of both the planktonic and benthic communities), we used measures of $\alpha, \beta$, and $\gamma$ based on the benthic macroinvertebrates and zooplankton taxa within each mesocosm. However, in the case of leaf litter decomposition, we used measures of $\alpha, \beta$, and $\gamma$ based only on non-predatory benthic macroinvertebrates because zooplankton do not consume benthic leaf litter (Shurin et al. 2012). First, we analyzed a single "global" model for each ecosystem 
function and tested for an interaction between each metric of diversity and environmental dissimilarity. A significant interaction would indicate the relationship between landscape level and ecosystem function differs with the level of environmental dissimilarity. After we found a significant $\beta$-environmental-dissimilarity interaction, we separately analyzed the relationship between each ecosystem function and $\alpha, \beta$, and $\gamma$ with data from each of the six landscapes with environmental dissimilarities of $0,3,4,6,7$, and 8 . These six separate analyses each produced a slope of the relationship between each ecosystem function and $\alpha, \beta$, or $\gamma$. As we were running six different regressions, we applied a Bonferroni correction (Weisstein 2004) to avoid inflating the chances of type 1 errors. This Bonferroni correction meant that we used an alpha level of 0.008 to determine significant differences. In order to understand the relative importance of $\alpha, \beta, \gamma$ and elevate temperature and nutrients, the $R^{2}$ value for the linear models was decomposed among factors using lmg metric from the calc.relimp function within the relaimpo package (Grömping 2006) in R. This metric decomposes the total $R^{2}$ value into non-negative portions for each variable that sums up to the total $R^{2}$ (Lindeman et al. 1980). As the order in which variables are added to a model can influence their relative importance, this metric takes the relative importance of each variable averaged over every order in which the variables can be added into the linear model (Lindeman et al. 1980, Johnson and LeBreton 2004, Grömping 2006).

\section{RESUlTS}

When $\alpha, \beta$, and $\gamma$ were measured in terms of their numbers equivalents (true diversity), landscape-level mean $\alpha$ did not have a significant relationship with the environmental dissimilarity index, but was reduced by $1.06 \pm 1.04$ (mean $\pm \mathrm{SE}$; $n=4845$ ) for every elevated temperature mesocosm. Mean $\alpha$ also decreased by $1.01 \pm 1.002$ for every elevated nutrient mesocosm within the landscape $\left(F_{1,4,841}=71.43, P<0.001\right)$. Landscape-level $\beta$ increased with increasing environmental heterogeneity, being $1.04 \pm 1.006$ (mean \pm SE) higher when

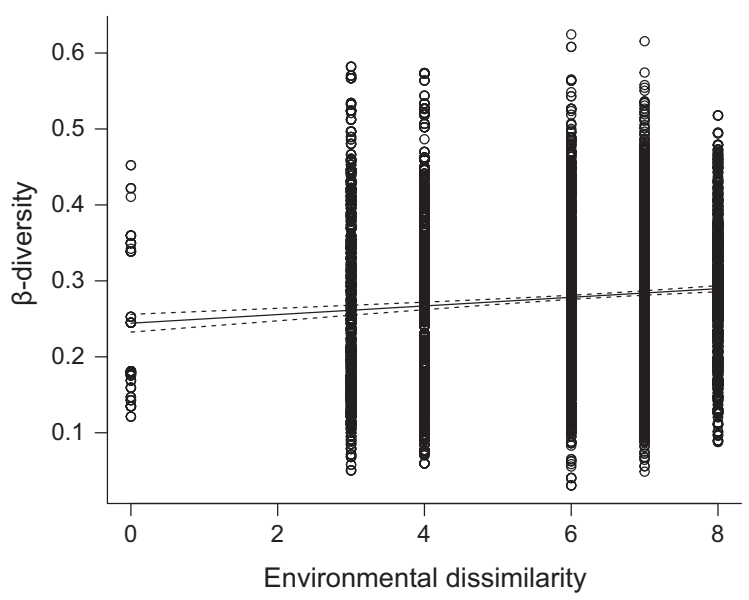

FIG. 1. $\beta$-diversity (measured as effective numbers) increases with increased environmental heterogeneity. Open circles represent raw data, solid line indicates fit of a linear regression model, and dashed lines indicate standard error estimates. the environmental dissimilarity index was 8 compared with 0 (linear regression, $F_{1,4,841}=161.22, P<0.001$, Fig. 1). However, we observed a wide range of $\beta$ across all levels of environmental dissimilarity, and there was substantial overlap of the ranges (Fig. 2). This high level of overlap in $\beta$ is critical to the following analyses, as it allows us to compare the relationship between $\beta$ and ecosystem functions across the different levels of environmental dissimilarity. In addition, $\beta$ increased by $1.03 \pm 1.0035$ for each mesocosm containing elevated nutrients $\left(F_{1,4,841}=1108.43, P<0.001\right)$ and increased by $1.03 \pm 1.003$ for each mesocosm with elevated temperature $\left(F_{1,4,841}=8.42, P<0.001\right)$. Landscape-level $\gamma$ increased with environmental dissimilarity $\left(F_{1,4,841}=99.21\right.$, $P<0.001)$, increased by $1.015 \pm 1.0025$ for every elevated nutrient mesocosm $\left(F_{1,4,841}=248.38, P<0.001\right)$ and decreased by $1.011 \pm 1.0023$ for each elevated temperature $\operatorname{mesocosm}\left(F_{1,4,841}=144.51, P<0.001\right)$.

To understand the relationships between ecosystem processes, diversity metrics and environmental conditions, we regressed both NEP and decomposition against $\alpha, \beta, \gamma$, and the number of mesocosms containing elevated temperature and nutrient treatments. Our global analysis of landscapelevel NEP revealed a significant interaction between environmental dissimilarity and $\beta$ (multiple linear regression, $\left.F_{1,4,835}=8.69, P=0.003\right)$. Specifically, the positive effect of $\beta$ on NEP and the amount of variation explained by $\beta$ increased as environmental dissimilarity increased (Fig. 2). NEP was associated with significant changes in $\alpha$ and $\gamma$ $(P<0.001)$, although neither diversity metric significantly interacted with environmental dissimilarity. NEP increased by $13.65 \% \pm 1.88 \%$ for each mesocosm containing elevated nutrients $\left(F_{1,4,835}=74.91, P<0.001\right)$ and increased by $2.75 \% \pm 0.63 \%$ for each elevated temperature mesocosm $\left(F_{1,4,835}=17.67, P<0.001\right)$. NEP significantly increased as mean $\alpha$ increased at all levels of environmental dissimilarity $\geq 3$ (Fig. 2A). Across all levels of environmental dissimilarity, the proportional change in NEP associated with $\alpha$ varied from 0.030 when environmental dissimilarity was 3 to 0.044 when environmental dissimilarity was 8 (Fig. 2B). NEP was involved in a significant positive relationship with $\beta$ at environmental dissimilarity levels $\geq 3$ (Fig. 2A) and accounted for between 0.034 and 0.080 of the variation in NEP (Fig. 2B). The relationships between NEP and $\gamma$ were positive when environmental dissimilarity was $\geq 3$ (Fig. 2A). The proportion of variation in NEP associated with $\gamma$ ranged between 0.037 and 0.060 (Fig. 2B). The relationship between NEP and nutrients was positive across all environmental dissimilarities where it could be assessed (level 8 could not be assessed) (Fig. 2A), and changes in the number of elevated nutrient mesocosms were associated with most of the proportional variation $(0.41-0.53$, Fig. 2 B). The relationship between NEP and temperature was positive across all levels of environmental dissimilarity where it could be assessed (Fig. 2A), and temperature accounted for between 0.010 and 0.017 of the proportional variation (Fig. 2B).

The global analysis revealed that landscape-level leaf litter decomposition was significantly associated with an interaction between $\beta$ and environmental dissimilarity $\left(F_{1,4,835}=\right.$ $32.12, P<0.001)$. The direction of this interaction indicated that the slope of the relationships between $\beta$ and decomposition increased with increasing environmental dissimilarity. 


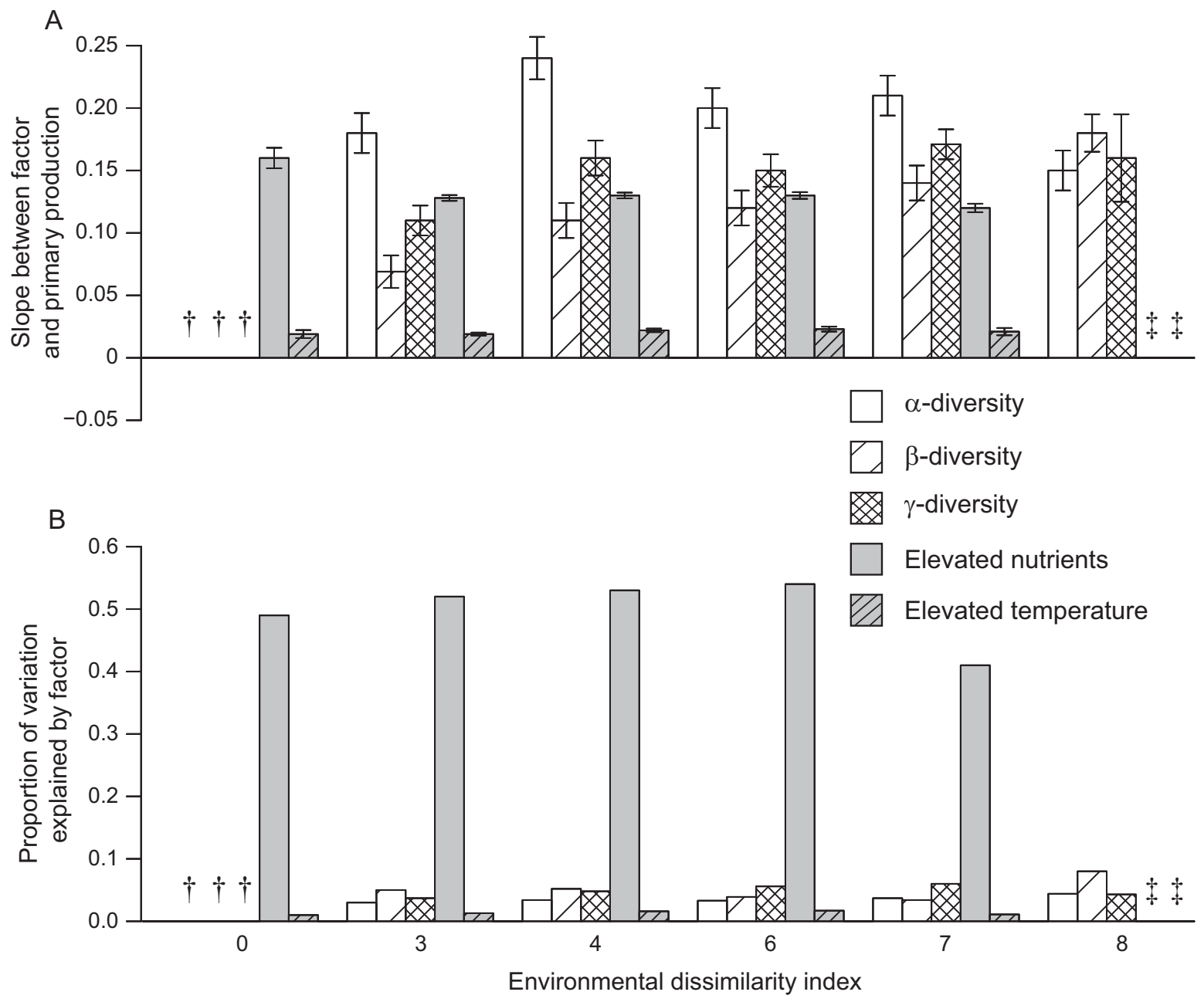

FIG. 2. Relationships between different metrics of biodiversity and environmental heterogeneity on rates of net ecosystem production. (A) Slope coefficients for each factor taken from a linear model incorporating all factors. (B) Proportion of the total variation in decomposition explained by each factor. A dagger $(\dagger)$ indicates a relationship was not significant $(P>0.008$ following a Bonferroni correction), and a double dagger $(\ddagger)$ indicates there was no variation in a factor, making a coefficient estimate impossible.

Decomposition rates varied with both $\alpha$ and $\gamma$ (both $P<0.001)$, although neither was involved in a significant interaction with environmental dissimilarity $(P=0.79$ and $P=0.23$, respectively). Decomposition rates also increased by $9.46 \% \pm 0.43 \%$ for each elevated nutrient mesocosm $\left(F_{1,4,835}=21.95, P<0.001\right)$ and increased by $13.02 \% \pm$ $0.50 \%$ for each elevated temperature mesocosm in the landscape $\left(F_{1,4,835}=26.20, P<0.001\right)$.

When landscapes with different environmental dissimilarity values were assessed separately, there was a positive relationship between decomposition and landscape $\alpha$ when environmental dissimilarity was 3 , a positive relationship when environmental dissimilarity was 8 , but no significant relationship when environmental dissimilarity was 0 , or 4-7 (Fig. 3A). The proportion of variation in decomposition explained by $\alpha$ was generally low (0.007-0.01 of the total variation, Fig. 3B). Our data showed a positive relationship between decomposition and $\beta$ at all levels of environmental dissimilarity other than 0 , and the slope coefficient of the relationship between decomposition and $\beta$ increased as the environmental dissimilarity index increased (Fig. 3A).
The proportion of variation in decomposition explained by $\beta$ ranged between 0.01 and 0.14 (Fig. 3B). Decomposition was positively associated with $\gamma$ at all levels of environmental dissimilarity (Fig. 3A), although the amount of variation accounted for by $\gamma$ decreased with increasing environmental dissimilarity from 0.27 to 0.06 (Fig. 3B). At levels of environmental dissimilarity where they could be assessed $(<8)$, decomposition rates were higher in landscapes with elevated nutrient and elevated temperature mesocosms (Fig. 3A). These environmental conditions accounted for the majority of the variation in decomposition (up to 0.35 for temperature at environmental dissimilarity $=0$ ), although the proportion they accounted for decreased as environmental dissimilarity increased (Fig. 3B).

Quantitatively similar relationships between environmental dissimilarity, diversity, and ecosystem functions were found for the analysis based on species richness rather than abundance-weighted metrics (Appendix S1). However, we found that when diversity measures were calculated using abundance-weighted metrics, they tended to account for slightly more (up to $0.1 \%$ ) of the variation in ecosystem 

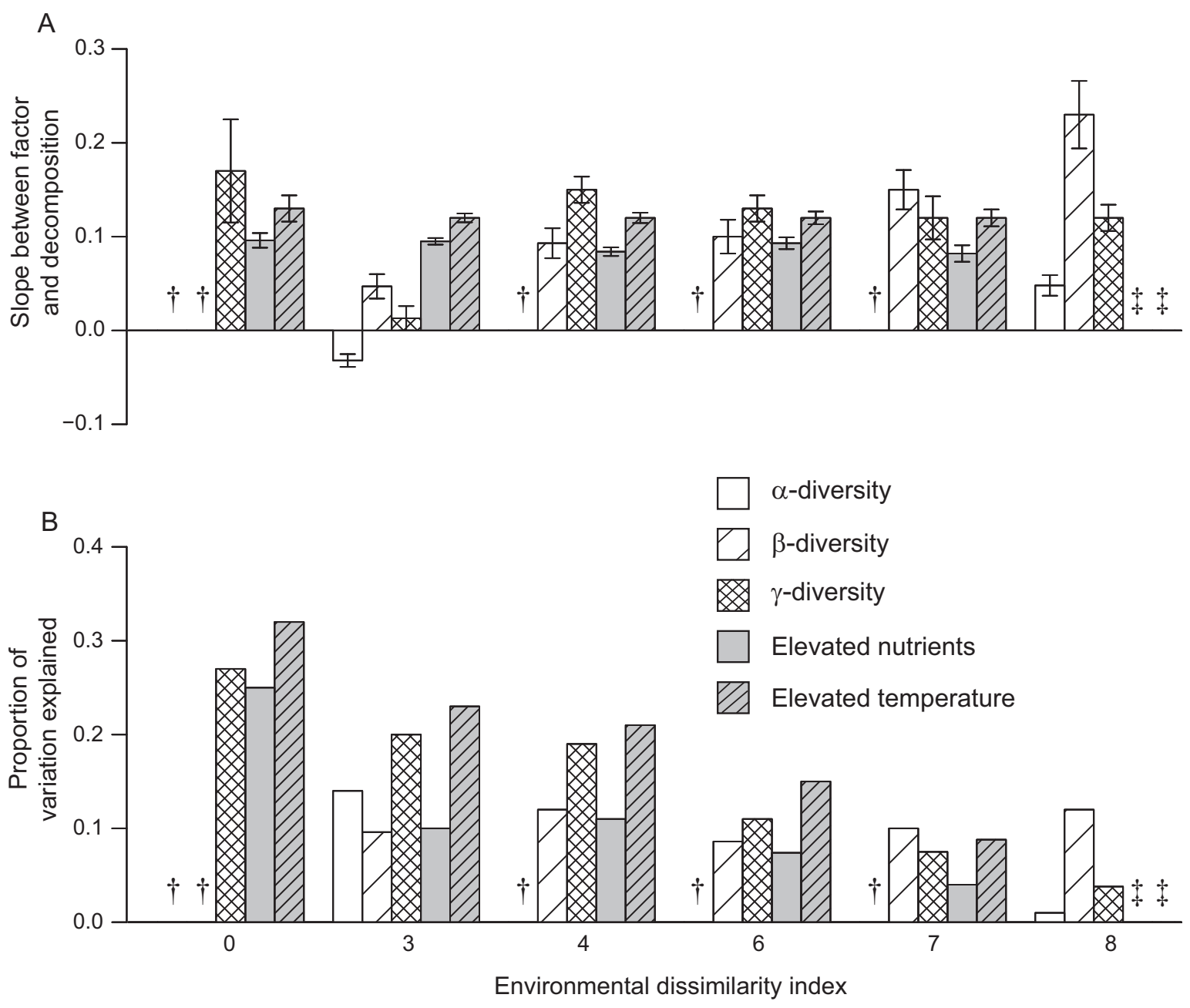

FIG. 3. Relationships between different metrics of biodiversity and environmental heterogeneity on rates of leaf litter decomposition. (A) Slope coefficients for each factor taken from a linear model incorporating all factors. (B) Proportion of the total variation in decomposition associated with each factor. A dagger $(\dagger)$ indicates a relationship was not significant $(P>0.008$ following a Bonferroni correction), and a double dagger $(\vdots)$ indicates there was no variation in a factor, making a coefficient estimate impossible.

function than when based on species richness. We believe this increase in variation may be linked to the extra information associated with including the numbers of individuals in abundance-weighted metrics and may also be linked to a pattern of decreasing species evenness with increasing environmental dissimilarity (Appendix S1: Fig. S4).

\section{Discussion}

We provide evidence that as landscape-level environmental heterogeneity increases, $\beta$ increases, which, in turn, is significantly associated with differences in landscape-level expressions of two ecosystem functions. In addition, while primary production and decomposition were primarily controlled by environmental conditions, at the highest levels of environmental dissimilarity $\beta$ appeared to explain a greater proportion of the variation in decomposition than either $\alpha$ or $\gamma$. This relatively large impact of $\beta$ compared to $\alpha$ or $\gamma$ suggests that in landscapes with a high level of spatial heterogeneity, the turnover of species among ecosystems is a more important driver of decomposition (a basal ecosystem process) than the actual composition of species. Relationships between biodiversity and ecosystem function have been investigated across a wide variety of systems (Tilman and Downing 1994, Bellwood et al. 2003, Girvan et al. 2005, Hattenschwiler et al. 2005, Atwood et al. 2015), but the majority of these previous studies focus on how biodiversity and community composition affect the processes within a specific ecosystem (Tilman and Downing 1994, Reich et al. 2005), or rates of flux to neighboring ecosystems (Atwood et al. 2014b). Recent experiments show that $\alpha, \beta$, and $\gamma$ can alter landscape-level patterns of ecosystem function (Pasari et al. 2013, Hautier et al. 2018, Winfree et al. 2018). Our results build on those studies by showing that the importance of $\beta$ as a driver of ecosystem functions may depend on the level of environmental dissimilarity among ecosystems in a landscape.

Our results revealed that as landscape-level environmental dissimilarity increased, landscape-level $\beta$ also increased, but we observed no concurrent changes in $\alpha$. This relationship between environmental dissimilarity and $\beta$ is consistent with ecological niche theory (species sorting), which proposes 
that different species are better adapted to different conditions (Vandermeer 1972, Chase and Leibold 2003), and aligns with recent findings from temperate forest (Barnes et al. 2016) and pollination systems (Winfree et al. 2018). Recent theory has shown that as environmental dissimilarity increases, increased $\beta$ becomes increasingly important for stabilizing ecological communities (Wang and Loreau 2014, 2016). The positive relationship we observed between environmental dissimilarity and $\beta$ may therefore mean that community stability is being maintained by increased species turnover as conditions across the landscape become more heterogeneous. In addition, $\beta$ and $\gamma$ increased as the number of elevated nutrient mesocosms in the landscape increased, while $\alpha$ decreased. Many studies have reported a unimodal, hump-shaped relationship between nutrient concentrations and biodiversity (Barnett and Beisner 2007, Cardinale et al. 2012b). Our finding of decreased $\alpha$ diversity with increased numbers of elevated nutrient landscapes suggests that nutrient levels in the experiment pass the threshold at which nutrients increase taxon dominance and reduce species richness (Nygaard and Ejrnæs 2009). The decrease in $\alpha$ associated with increased temperature suggests that this abiotic factor may represent a stressor on the ecosystem, reducing the diversity present. The increase in $\beta$ associated with elevated nutrients and temperature suggests that as nutrients and temperature increase, stochastic determinants of community composition such as priority effects become stronger (Chase 2010), enhancing dissimilarity among ecosystems.

The positive relationships between NEP and $\alpha$ and $\beta$ at environmental dissimilarity greater than 0 would suggest that high primary production is providing a resource that can lead to high consumer diversity (Tilman et al. 1982, Chase and Leibold 2002). In addition, the greatest driver of primary production within the experiment was the number of elevated nutrient mesocosms within a landscape. Previous continental scale studies have also demonstrated that the majority of variation in primary productivity is driven by abiotic conditions, with diversity contributing far less (Burley et al. 2016). The combination of the positive relationship between $\alpha$ and $\beta$ and primary production together with the high proportion of variation associated with elevated nutrients would suggest that, in our model system, primary production and the invertebrate consumer community it supports are strongly influenced by bottom-up processes.

When environmental dissimilarity was 0 , rates of leaf litter decomposition were not associated with either $\alpha$ or $\beta$, where positively influenced by $\gamma$, but primarily controlled by elevated temperature and nutrients. The positive effect of elevated temperature on decomposition rates likely stems from higher temperatures increasing rates of microbial metabolism (Geraldes et al. 2012, Follstad Shah et al. 2017). The positive effects of elevated nutrients on decomposition may have occurred through a priming effect, as increased primary producer biomass leads to the production of algal-based carbon exudates that can stimulate bacterial growth (Danger et al. 2016). Unlike primary production, which can be influenced by both consumption and algal growth, litter standing stocks can only be influenced by consumption as there was no mechanism by which leaf litter could be added. This lack of an addition mechanism could potentially explain why diversity had a stronger general impact on decomposition than primary productivity, as elevated nutrients are likely to promote algal growth and counteract the effects of consumption.

Our study provides compelling evidence that $\beta$-diversity may increase ecosystem functions across heterogeneous landscapes. Previous investigations using mesocosms observed that increased $\beta$ generally did not affect mean levels of ecosystem function in landscapes with similar abiotic conditions, although it did substantially increase the variance in rates of functional processes (Pasari et al. 2013). This previous observation is consistent with our results in that we found no significant relationship between $\beta$ and either decomposition or primary production when all ecosystems within a landscape had the same environmental conditions. However, increased environmental dissimilarity led to both increased $\beta$ and an increase in the strength of the relationship between $\beta$ and ecosystem functions, to the extent that $\beta$ was more strongly associated with ecosystem functions than either $\alpha$ or $\gamma$. The relatively high importance of $\beta$ as environmental dissimilarity increases agrees with previous field experiments investigating the relationships between diversity and ecosystem functions (Isbell et al. 2017, Winfree et al. 2018). These previous investigations specifically note that as the spatial scale over which ecosystem functions are measured increases, environmental conditions are likely to become increasingly heterogeneous, meaning different species are better suited to performing different functions (Winfree et al. 2018). As anthropogenic stressors affect some ecosystems more than others (Ashcroft et al. 2009) and can homogenize communities (Mondy and Usseglio-Polatera 2014), understanding the relationships between diversity, environmental heterogeneity and ecosystem functions is crucial to estimating the wider impact of human alteration of landscapes.

\section{ACKNOWLEDGMenTs}

We thank Annalise Barber, Andrew O'Farrell, Dylan Rawlyk, Matt Robinson, Anne Rutherford, and Patrick Thompson for assistance in the laboratory and field. This work would not have been possible without water heaters provided by Wendy Palen and Dolph Schluter's assistance with the UBC experimental pond facility.

\section{Literature Cited}

Ashcroft, M. B., L. A. Chisholm, and K. O. French. 2009. Climate change at the landscape scale: predicting fine-grained spatial heterogeneity in warming and potential refugia for vegetation. Global Change Biology 15:656-667.

Atwood, T. B., E. Hammill, and J. S. Richardson. 2014a. Trophiclevel dependent effects on $\mathrm{CO}_{2}$ emissions from experimental stream ecosystems. Global Change Biology 20:3386-3396.

Atwood, T. B., E. Hammill, D. S. Srivastava, and J. S. Richardson. 2014b. Competitive displacement alters top-down effects on carbon dioxide concentrations in a freshwater ecosystem. Oecologia 175:353-361.

Atwood, T. B., R. M. Connolly, E. G. Ritchie, C. E. Lovelock, M. R. Heithaus, G. C. Hays, J. W. Fourqurean, and P. I. Macreadie. 2015. Predators help protect carbon stocks in blue carbon ecosystems. Nature Climate Change 5:1038-1045.

Bailey, R. C., R. H. Norris, and T. B. Reynoldson. 2004. Bioassessment of freshwater ecosystems. Springer, Boston, Massachusetts, USA. 
Barbour, M. T., J. Gerritsen, B. D. Snyder, and J. B. Stribling. 1999. Rapid bioassessment protocols for use in streams and wadeable rivers. USEPA, Washington, D.C., USA.

Barnes, A. D., P. Weigelt, M. Jochum, D. Ott, D. Hodapp, N. F. Haneda, and U. Brose. 2016. Species richness and biomass explain spatial turnover in ecosystem functioning across tropical and temperate ecosystems. Philosophical Transactions of the Royal Society B 371:20150279.

Barnett, A., and B. E. Beisner. 2007. Zooplankton biodiversity and lake trophic state: Explanations invoking resource abundance and distribution. Ecology 88:1675-1686.

Barwell, L. J., N. J. B. Isaac, and W. E. Kunin. 2015. Measuring $\beta$ diversity with species abundance data. Journal of Animal Ecology 84:1112-1122.

Beck, J., J. D. Holloway, and W. Schwanghart. 2013. Undersampling and the measurement of beta diversity. Methods in Ecology and Evolution 4:370-382.

Bellwood, D. R., A. S. Hoey, and J. H. Choat. 2003. Limited functional redundancy in high diversity systems: resilience and ecosystem function on coral reefs. Ecology Letters 6:281-285.

Bouwman, A. F., A. H. W. Beusen, and G. Billen. 2009. Human alteration of the global nitrogen and phosphorus soil balances for the period 1970-2050. Global Biogeochemical Cycles 23: GB0A04

Burley, H. M., K. Mokany, S. Ferrier, S. W. Laffan, K. J. Williams, and T. D. Harwood. 2016. Primary productivity is weakly related to floristic alpha and beta diversity across Australia. Global Ecology and Biogeography 25:1294-1307.

Cardinale, B. J., D. S. Srivastava, J. E. Duffy, J. P. Wright, A. L. Downing, M. Sankaran, and C. Jouseau. 2006. Effects of biodiversity on the functioning of trophic groups and ecosystems. Nature 443:989-992.

Cardinale, B. J., et al. 2012a. Biodiversity loss and its impact on humanity. Nature 489:326.

Cardinale, J., D. M. Bennett, E. Nelson, and K. Gross. 2012b. Does productivity drive diversity or vice versa? A test of the multivariate productivity-diversity hypothesis in streams. Ecology 90:1227-1241.

Chase, J. M. 2010. Stochastic community assembly causes higher biodiversity in more productive environments. Science 328:13881391.

Chase, J. M., and M. A. Leibold. 2002. Spatial scale dictates the productivity-biodiversity relationship. Nature 416:427-430.

Chase, J. M., and M. A. Leibold. 2003. Ecological niches: linking classical and contemporary approaches. University of Chicago Press, Chicago, Illinois, USA.

Danger, M., J. Cornut, E. Chauvet, P. Chavez, and A. Elger. 2016. Benthic algae stimulate leaf litter decomposition in detritus-based headwater streams: a case of aquatic priming effect? Ecology 94:1604-1613.

Dupas, R., M. Delmas, J.-M. Dorioz, J. Garnier, F. Moatar, and C. Gascuel-Odoux. 2015. Assessing the impact of agricultural pressures on $\mathrm{N}$ and $\mathrm{P}$ loads and eutrophication risk. Ecological Indicators 48:396-407.

Follstad Shah, J. J., et al. 2017. Global synthesis of the temperature sensitivity of leaf litter breakdown in streams and rivers. Global Change Biology 23:3064-3075.

Forster, P., et al. 2007. Changes in atmospheric constituents and in radiative forcing. Chapter 2. Climate change 2007. The physical science basis. Cambridge University Press, Cambridge, UK.

Geraldes, P., C. Pascoal, and F. Cássio. 2012. Effects of increased temperature and aquatic fungal diversity on litter decomposition. Fungal Ecology 5:734-740.

Girvan, M. S., C. D. Campbell, K. Killham, J. I. Prosser, and L. A. Glover. 2005. Bacterial diversity promotes community stability and functional resilience after perturbation. Environmental Microbiology 7:301-313.

Greig, H. S., P. Kratina, P. L. Thompson, W. J. Palen, J. S. Richardson, and J. B. Shurin. 2012. Warming, eutrophication, and predator loss amplify subsidies between aquatic and terrestrial ecosystems. Global Change Biology 18:504-514.
Grime, J. P. 1998. Benefits of plant diversity to ecosystems: Immediate, filter and founder effects. Journal of Ecology 86:902-910.

Grömping, U. 2006. R package relaimpo: relative importance for linear regression. Journal of Statistical Software 17:139-147.

Gruber, N., and J. N. Galloway. 2008. An Earth-system perspective of the global nitrogen cycle. Nature 451:293-296.

Harpole, W. S., et al. 2011. Nutrient co-limitation of primary producer communities. Ecology Letters 14:852-862.

Hattenschwiler, S., A. V. Tiunov, and S. Scheu. 2005. Biodiversity and litter decomposition in terrestrial ecosystems. Annual Review of Ecology, Evolution and Systematics 36:191-218.

Hautier, Y., et al. 2018. Local loss and spatial homogenization of plant diversity reduce ecosystem multifunctionality. Nature Ecology \& Evolution 2:50-56.

Heathwaite, A. L., P. F. Quinn, and C. J. M. Hewett. 2005. Modelling and managing critical source areas of diffuse pollution from agricultural land using flow connectivity simulation. Journal of Hydrology 304:446-461.

Hobbs, R. J., E. Higgs, and J. A. Harris. 2009. Novel ecosystems: implications for conservation and restoration. Trends in Ecology \& Evolution 24:599-605.

Isbell, F., et al. 2017. Linking the influence and dependence of people on biodiversity across scales. Nature 546:65-72.

Johnson, J. W., and J. M. LeBreton. 2004. History and use of relative importance indices in organizational research. Organizational Research Methods 7:238-257.

Jost, L. 2007. Partitioning diversity into independent alpha and beta components. Ecology 88:2427-2439.

Jost, L. 2010. Independence of alpha and beta diversity. Ecology 91:1969-1974.

Kraft, N. J. B., R. Valencia, and D. D. Ackerly. 2008. Functional traits and niche-based tree community assembly in an Amazonian forest. Science 322:580-582.

Kratina, P., H. S. Greig, P. L. Thompson, T. S. A. Carvalho-Pereira, and J. B. Shurin. 2012. Warming modifies trophic cascades and eutrophication in experimental freshwater communities. Ecology 93:1421-1430

Lefcheck, J. S., J. E. K. Byrnes, F. Isbell, L. Gamfeldt, J. N. Griffin, N. Eisenhauer, M. J. S. Hensel, A. Hector, B. J. Cardinale, and J. E. Duffy. 2015. Biodiversity enhances ecosystem multifunctionality across trophic levels and habitats. Nature Communications 6:1-7.

Lindeman, R. H., P. F. Merenda, and R. Z. Gold. 1980. Introduction to bivariate and multivariate analysis. Foresman and Company, London, UK.

Loreau, M., S. Naeem, P. Inchausti, J. Bengtsson, J. P. Grime, A. Hector, D. U. Hooper, M. A. Huston, D. Raffaelli, and B. Schmid. 2001. Biodiversity and ecosystem functioning: current knowledge and future challenges. Science 294:804-808.

Marcon, E., and B. Hérault. 2015. entropart: An R package to measure and partition diversity. Journal of Statistical Software 67:1-26.

Mondy, C. P., and P. Usseglio-Polatera. 2014. Using fuzzy-coded traits to elucidate the non-random role of anthropogenic stress in the functional homogenisation of invertebrate assemblages. Freshwater Biology 59:584-600.

Naeem, S., J. E. Duffy, and E. Zavaleta. 2012. The functions of biological diversity in an age of extinction. Science 336:1401-1406.

Nygaard, B., and R. Ejrnæs. 2009. The impact of hydrology and nutrients on species composition and richness: Evidence from a microcosm experiment. Wetlands 29:187-195.

Okubo, A., and S. A. Levin. 2013. Diffusion and ecological problems: modern perspectives. Springer-Verlag, New York City, New York, USA.

Pasari, J. R., T. Levi, E. S. Zavaleta, and D. Tilman. 2013. Several scales of biodiversity affect ecosystem multifunctionality. Proceedings of the National Academy of Sciences USA 110:1021910222.

Reich, P. B., J. Oleksyn, J. Modrzynski, P. Mrozinski, S. E. Hobbie, D. M. Eissenstat, J. Chorover, O. A. Chadwick, C. M. Hale, and M. G. Tjoelker. 2005. Linking litter calcium, earthworms and soil 
properties: a common garden test with 14 tree species. Ecology Letters 8:811-818.

Resh, V. H. 2007. Multinational, freshwater biomonitoring programs in the developing world: lessons learned from African and Southeast Asian river surveys. Environmental Management 39:737-748.

Shurin, J. B., J. L. Clasen, H. S. Greig, P. Kratina, and P. L. Thompson. 2012. Warming shifts top-down and bottom-up control of pond food web structure and function. Philosophical Transactions of the Royal Society B 367:3008-3017.

Thompson, P. L., and A. Gonzalez. 2016. Ecosystem multifunctionality in metacommunities. Ecology 97:2867-2879.

Tilman, D. 1999. Global environmental impacts of agricultural expansion: the need for sustainable and efficient practices. Proceedings of the National Academy of Sciences USA 96:5995-6000.

Tilman, D., and J. A. Downing. 1994. Biodivesity and stability in grasslands. Nature 367:363-365.

Tilman, D., S. S. Kilham, and P. Kilham. 1982. Phytoplankton community ecology: The role of limiting nutrients. Annual Review of Ecology and Systematics 13:349-372.

Vandermeer, J. 1972. Niche theory. Annual Review of Ecology and Systematics 3:107-132.

Vitousek, P. M., H. A. Mooney, J. Lubchenco, and J. M. Melillo. 1997. Human domination of earth ecosystems. Science 277:464 499.
Wang, S., and M. Loreau. 2014. Ecosystem stability in space: $\alpha, \beta$ and $\gamma$ variability. Ecology Letters 17:891-901.

Wang, S., and M. Loreau. 2016. Biodiversity and ecosystem stability across scales in metacommunities. Ecology Letters 19:510-518.

Wang, Z., J. H. Brown, Z. Tang, and J. Fang. 2009. Temperature dependence, spatial scale, and tree species diversity in eastern Asia and North America. Proceedings of the National Academy of Sciences USA 106:13388-13392.

Weisstein, E. W. 2004. Bonferroni correction. Wolfram Research. http://mathworld.wolfram.com/BonferroniCorrection.html

Whittaker, R. H. 1972. Evolution and measurement of species diversity. Taxon 12:213-251.

Winder, M., J. E. Reuter, and S. G. Schladow. 2009. Lake warming favours small-sized planktonic diatom species. Proceedings of the Royal Society B 276:427-435.

Winfree, R., J. R. Reilly, I. Bartomeus, D. P. Cariveau, N. M. Williams, and J. Gibbs. 2018. Species turnover promotes the importance of bee diversity for crop pollination at regional scales. Science 359:791-793.

Worm, B., H. K. Lotze, H. Hillebrand, and U. Sommer. 2002. Consumer versus resource control of species diversity and ecosystem functioning. Nature 417:848-851.

SUPPORTING INFORMATION

Additional supporting information may be found in the online version of this article at http://onlinelibrary.wiley.com/doi/10.1002/ecy. 2492/suppinfo

\section{Data Availability}

Data are available from the Dryad Digital Repository: https://doi.org/10.5061/dryad.8180ph5 